Research Article

\title{
Score and Correlation Coefficient-Based Feature Selection for Predicting Heart Failure Diagnosis by Using Machine Learning Algorithms
}

\author{
Ebrahim Mohammed Senan $\left(\mathbb{D},{ }^{1}\right.$ Ibrahim Abunadi $\left(\mathbb{D},{ }^{2}\right.$ Mukti E. Jadhav $\mathbb{D}^{3}$ \\ and Suliman Mohamed Fati ${ }^{2}{ }^{2}$ \\ ${ }^{1}$ Department of Computer Science \& Information Technology, Dr. Babasaheb Ambedkar Marathwada University, \\ Aurangabad, India \\ ${ }^{2}$ Information Systems Department, Prince Sultan University, Riyadh, Saudi Arabia \\ ${ }^{3}$ Shri Shivaji Science \& Arts College, Chikhli Dist., Buldana, India \\ Correspondence should be addressed to Ebrahim Mohammed Senan; senan1710@gmail.com
}

Received 19 September 2021; Revised 20 November 2021; Accepted 3 December 2021; Published 20 December 2021

Academic Editor: Jose Joaquin Rieta

\begin{abstract}
Copyright (c) 2021 Ebrahim Mohammed Senan et al. This is an open access article distributed under the Creative Commons Attribution License, which permits unrestricted use, distribution, and reproduction in any medium, provided the original work is properly cited.
\end{abstract}

\begin{abstract}
Cardiovascular disease (CVD) is one of the most common causes of death that kills approximately 17 million people annually. The main reasons behind CVD are myocardial infarction and the failure of the heart to pump blood normally. Doctors could diagnose heart failure (HF) through electronic medical records on the basis of patient's symptoms and clinical laboratory investigations. However, accurate diagnosis of HF requires medical resources and expert practitioners that are not always available, thus making the diagnosing challengeable. Therefore, predicting the patients' condition by using machine learning algorithms is a necessity to save time and efforts. This paper proposed a machine-learning-based approach that distinguishes the most important correlated features amongst patients' electronic clinical records. The SelectKBest function was applied with chi-squared statistical method to determine the most important features, and then feature engineering method has been applied to create new features correlated strongly in order to train machine learning models and obtain promising results. Optimised hyperparameter classification algorithms SVM, KNN, Decision Tree, Random Forest, and Logistic Regression were used to train two different datasets. The first dataset, called Cleveland, consisted of 303 records. The second dataset, which was used for predicting HF, consisted of 299 records. Experimental results showed that the Random Forest algorithm achieved accuracy, precision, recall, and F1 scores of 95\%, 97.62\%, 95.35\%, and 96.47\%, respectively, during the test phase for the second dataset. The same algorithm achieved accuracy scores of $100 \%$ for the first dataset and $97.68 \%$ for the second dataset, while $100 \%$ precision, recall, and F1 scores were reached for both datasets.
\end{abstract}

\section{Introduction}

Cardiovascular disease (CVD) is one of the most common diseases that cause morbidity and mortality. It contributes to a third of deaths worldwide according to the American College [1]. Since 2102, numerous surveys concluded that nearly 56 million people lost their lives in 2012; amongst them, 17.5 million died due to CVD [2]. According to [3], CVD has three types: circulatory, structural, and electrical. In circulatory CVD, which is also called coronary artery disease (CAD), atherosclerosis (i.e., accumulation of plaques) is built up on the inner walls of a coronary artery, causing the arteries to harden [4-6]. This accumulated plaque consists of cholesterol or fatty deposits that restrict blood flow through the arteries. When CAD progresses, potentially fatal symptoms, such as stroke and myocardial infarction, begin to appear.

Therefore, early treatment and recovery of atherosclerosis are important to minimise CVD risks. Several imaging methods are introduced with high accuracy and sensitivity 
to determine the disease severity $[7,8]$. They include dobutamine stress echocardiography, exercise electrocardiogram (ECG), coronary computed tomography angiography, myocardial perfusion scintigraphy, and conventional coronary angiography. However, all these imaging methods could only discover existing atherosclerosis that already developed. For instance, ECG is an easy-to-access diagnostic tool that records the electrical activity of the heart [9]. ECG signals could be obtained during exercise as a patient undergoes stress [10]. ECG signals allow heart rate variability signals to be extracted [11]. The ECG technique is the primary choice for evaluating heart conditions because it is easy to perform, inexpensive, and noninvasive. However, manual diagnosis of ECG signals is tedious and difficult because the signals differ morphologically.

In addition, the discovery of biomarkers, such as chest pain, serum cholesterol, resting electrocardiographic results, resting blood pressure, maximum heart rate, depression, fasting blood sugar, exercise-induced angina, slope of peak exercise, number of major vessels, segment, and thallium stress, in clinical samples has been helpful in understanding and diagnosing atherosclerosis. Therefore, to conduct a diagnostic process of ECG signals with high accuracy, artificial intelligence techniques are used to help diagnose arteriosclerosis through these tests and biomarkers. As the data collected for these biomarkers are huge, most of the studies on the diagnosis systems focused on the preprocessing process to clean the data, select the most important representative features, delete redundant features, and choose appropriate classification algorithms. For instance, Zimmerli et al. presented an assay for polypeptides that contribute to biomarkers for identifying CAD. They screened 359 urine samples from 88 patients with CAD and 282 controls. The system reached a sensitivity of $98 \%$ and a specificity of $83 \%$ [12]. Likewise, Tan et al. presented three diagnostic algorithms for a set of diagnostic features of heart disease. The systems were evaluated by accuracy, sensitivity, and specificity on four datasets: Cleveland, Hungarian, SPECTF, and Switzerland. Their proposed system reached accuracy scores of $81.19 \%, 92.68 \%, 82.7 \%$, and $84.52 \%$ for Cleveland, Hungarian, SPECTF, and Switzerland, respectively [13]. Arabasadi et al. presented a hybrid method to diagnose $\mathrm{CAD}$, and their algorithm was able to increase the performance of neural networks by $10 \%$ through a genetic algorithm (GA), which optimises primary weights. The system achieved an accuracy of $93.85 \%$, a sensitivity of $97 \%$, and a specificity of $92 \%$ [14]. Maji and Arora presented a hybrid method between Decision Tree and ANN classifiers for diagnosing heart disease. The ANN achieved an accuracy of $77.4 \%$, a sensitivity of $77.4 \%$, and a specificity of $21.7 \%$ [15]. Saqlain et al. presented three algorithms for selecting the most important features, which are the Fisher scorebased algorithm, the algorithm for selecting the most important features based on forward, and the algorithm for selecting the most important features based on the reverse. The selected features were entered into an SVM classifier based on the RBF kernel for the diagnosis of four cardiac disease datasets. The system achieved an accuracy of $81.19 \%$ for the Cleveland dataset [16]. Babu et al. applied
14 features that were extracted, then fed into three classification algorithms, namely, $K$-means, MAFI, and Decision Tree, to classify heart disease. All algorithms performed well for diagnosing heart failure [17]. Reddy and Khare presented a rule-based fuzzy logic (RBFL) algorithm to predict heart disease and help medical practitioners diagnose it at an early stage. The locality preserving projection (LPP) method was first applied to determine the most important characteristics of the UCI dataset. The RBFL algorithm achieved an accuracy of $78 \%$ [18]. Feshki et al. presented Particle Swarm Optimization method with Neural Network Feed Forward Back Propagation, which reduced the features from 13 features to 8 enhanced features; the system reached an accuracy of $91.94 \%$ with these selected features [19]. Uyar and İlhan presented a GA based on a Recurrent Fuzzy Neural Network (RFNN) algorithm trained to diagnose heart disease, and the system achieved an accuracy of $97.78 \%$ [20]. Haq et al. presented seven machine learning algorithms to classify features extracted by three methods for selecting features of the heart failure dataset. The performance of the systems was evaluated using several scales such as accuracy, sensitivity, specificity, receiving optimism curves, and AUC, and they reached good results [21]. Kerexeta et al. presented two methods for predicting the risk of returning a patient with high blood pressure back to hospital. In the first method, the supervised and supervised classification methods were combined, and the system reached an AUC of $61 \%$. The second method was combined the Naïve Bayes classifiers and the Decision Tree, and the method achieved an AUC of $73 \%$. The limitations in this study are related to the dataset because the study is based on a readmission day threshold [22]. Adler et al. presented machine learning algorithms that link patient features with mortality, by training a Decision Tree algorithm with a set of features associated with high mortality risk. Eight characteristics that have a very high risk of death were extracted, and the risk score for these advantages was $88 \%$ for the AUC scale. Limitation of MARKERHF was derived from two hospitals, San Diego, California, and is therefore subject to demographic region bias [23]. Jin et al. presented an effective method for predicting heart failure by using a neural network, where they used one-hot encoding and word vectors to model the diagnosis and prediction of heart failure through a long short-term memory algorithm [24]. Gjoreski et al. presented a method that combines machine learning and deep learning to diagnose heart failure based on the heart sounds of 947 people. Machine learning algorithms train expert features, while deep learning models train from the spectral chains of the heart signal. The method achieved an accuracy of 92.9 and an error rate of $7.1 \%$ [25]. Vijayashree and Sultana presented the Particle Swarm Optimization (PSO) method, which selects the most appropriate features and increases the important features for diagnosing heart disease. PSO was used in conjunction with SVM to reduce the number of features and increase accuracy; the system achieved good results for diagnosing heart disease [26].

However, most of the discussed studies above are insufficient. Therefore, the main contributions of this paper are as follows: 
(i) Adjust and optimise hyperparameter of five machine learning algorithms for predicting heart failure (HF) with high accuracy

(ii) Select the most important features with strong correlation to obtain more realistic diagnostic results

(iii) Apply feature scoring to rank the features based on the correlated to the target feature

(iv) Solve the class imbalance issue in the second dataset by synthetic minority oversampling (SMOTE) technique

(v) Create new features that have strong correlation with the target feature to obtain more realistic diagnostic results

The remainder of this study is organised as follows. Section 2 describes a background on the overview and risk factors of HF diseases and an explanation of machine learning algorithms. Section 3 discusses the exploratory data analysis (EDA) to describe the two sets of data and explain the correlation between the features and the replacement of missing values. Section 4 presents data processing that includes subsections for engineering and selection of the most important features. Section 5 describes the experimental result and discussion part. Finally, Section 6 concludes the paper.

\section{Background}

2.1. Overview and Risk Factors of HF Diseases. Heart disease and atherosclerosis are disorders of the heart and arteries that include HF, coronary heart disease (heart attacks), cerebrovascular diseases (strokes), and other types of heart disease [27]. CVD is one of the most common causes of death in the world, with the number of deaths reaching roughly 17 million annually worldwide. HF occurs because the heart is unable to pump enough blood to the rest of the body. It is caused by diabetes, high blood pressure, and other heart diseases [28]. Doctors classify HF into two types on the basis of the ejection fraction value, which is the percentage of blood that the heart pumps during one contraction and a physiological value ranging from $50 \%$ to $75 \%$. Low HF causes the ejection fraction, previously called left ventricular (LV) HF, to drop below 40\% [29]. The final ejection fraction rate is $\mathrm{HF}$ with preserved ejection fraction, previously called diastolic HF, with a normal ejection fraction. In this case, during systole, the LV contracts normally but fails during diastole due to ventricular stiffness; thus, blood pumping is impaired [30]. Due to the importance of the heart organ, HF prediction has become of utmost importance for physicians in predicting HF; however, even today in clinical practices, physicians have failed to reach high accuracy in predicting HF [31]. Electronic medical records could be considered one of the most useful sources for uncovering correlated data amongst patients and an important source for researchers and clinical practices [32]. Machine learning techniques play an important role in analysing medical records, predicting the survival of each patient with

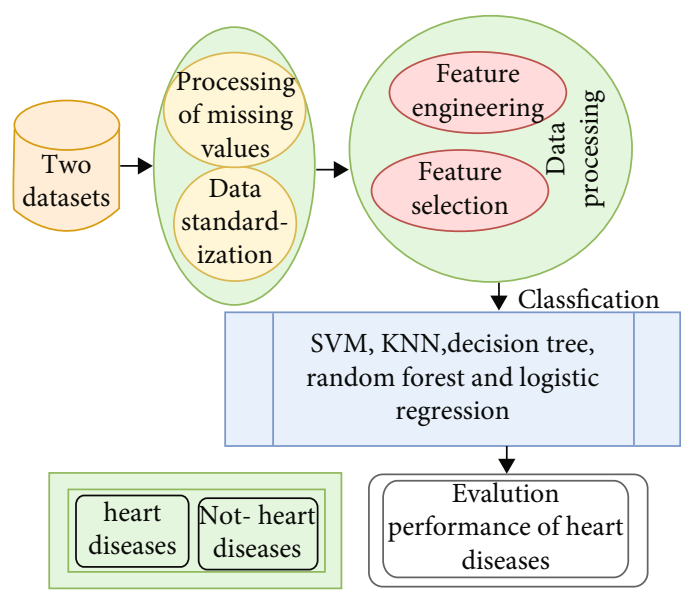

FIGURE 1: Experimental methodology of heart disease.

$\mathrm{HF}$, and detecting the most important features that lead to HF [33].

2.2. Machine Learning. Machine learning is the ability of computer programs to adapt, learn, and address new problems. Machine learning algorithms work on medical diagnostics and help experts support their decisions about their medical diagnosis. Machine learning has the ability to learn from training data and solve classification problems for new data [34].

2.2.1. K-Nearest Neighbor (KNN). KNN is used to solve classification problems based on stored data. The algorithm trains the dataset and stores it in the memory. When the classification process is to test new data points, the algorithm works on the basis of similarity of the state between the new data point and the stored dataset and classifies new data in accordance with the most similar class on the basis of the value of $K$ and the closest one on the basis of Euclidean distance.

2.2.2. Support Vector Machine (SVM). This model is similar to neural networks in its objective of adjusting a set of parameters, which allow to establish boundaries in a dimensional space and approximate functions or separate patterns in different regions of the attribute space. The difference lies in the training method for adjusting the parameters. By contrast, SVMs base their training on maximizing the margin between the hyperplane and the instances of two classes (initially, this model was designed to solve problems of classifying two classes but extensions for multiclass and regression problems exist) [35]. The algorithm works with linear and nonlinear data. When the data are linear, the algorithm finds a hyperplane with maximum margin, which is the largest distance between data points of two classes. Maximum margin gives the algorithm power to classify the test dataset with high confidence. Hyperplane is the decision boundary that separates the class data. Support vectors are the data points that form close to the hyperplane. In accordance with support vectors, the distance is increased to maximize the margin. Thus, the hyperplanes change 
TABle 1: Diagnosing heart disease features with metrics from the Cleveland dataset.

\begin{tabular}{|c|c|c|c|}
\hline Features & Description & Explanation & Type \\
\hline Age & Patient age & Age of patient in year & Numeric \\
\hline \multirow{2}{*}{ Sex } & \multirow{3}{*}{ Patient gender } & $1=$ male & \multirow{2}{*}{ Nominal } \\
\hline & & $0=$ female & \\
\hline \multirow{4}{*}{$\mathrm{cp}$} & & $1=$ typical angina & \multirow{4}{*}{ Nominal } \\
\hline & Chest pain & $2=$ atypical angina & \\
\hline & & $3=$ nonanginal pain & \\
\hline & & $4=$ asymptomatic & \\
\hline trestbps & Patient's blood pressure at rest $(\mathrm{mm} / \mathrm{Hg})$ & Resting blood pressure $(\mathrm{mm} / \mathrm{Hg})$ & Numeric \\
\hline chol & Patient's cholesterol (mg/dL) & Serum cholesterol (mg/dL) & Numeric \\
\hline \multirow{2}{*}{$\mathrm{fbs}$} & & $1=$ Fasting blood sugar $>120 \mathrm{mg} / \mathrm{dL}$ & \multirow{2}{*}{ Nominal } \\
\hline & Patient's blood sugar during fasting & $0=$ Fasting blood sugar $<120 \mathrm{mg} / \mathrm{dL}$ & \\
\hline \multirow{3}{*}{ restecg } & & $0=$ normal & \multirow{3}{*}{ Nominal } \\
\hline & Electrocardiographic measurement at rest & $1=\mathrm{ST}-\mathrm{T}$ wave abnormality & \\
\hline & & $2=$ probable left ventricular hypertrophy & \\
\hline thalach & Maximum heart rates & Maximum heart rate achieved & Numeric \\
\hline \multirow{2}{*}{ exang } & Angina due to exercise & $1=$ exercise induced angina & \multirow{2}{*}{ Nominal } \\
\hline & & $0=$ exercise induced no angina & \\
\hline \multirow[t]{2}{*}{ Oldpeak } & ST depression & ST depression induced by exercise relative to rest & \multirow[t]{2}{*}{ Numeric } \\
\hline & & $1=$ upsloping & \\
\hline \multirow[t]{2}{*}{ Slope } & Slope of ST & $2=$ flat & \multirow[t]{2}{*}{ Nominal } \\
\hline & & $3=$ downsloping & \\
\hline \multirow[t]{2}{*}{$\mathrm{ca}$} & Number of major vessels & Number of major vessels $(0-3)$ colored by fluoroscopy & \multirow[t]{2}{*}{ Numeric } \\
\hline & & $3=$ normal & \\
\hline \multirow[t]{2}{*}{ thal } & Blood disorder & $6=$ fixed defect & \multirow[t]{2}{*}{ Nominal } \\
\hline & & $7=$ reversible defect & \\
\hline \multirow{2}{*}{ Target } & & $0=$ normal & \multirow[t]{2}{*}{ Nominal } \\
\hline & & $1=$ heart disease & \\
\hline
\end{tabular}

when removing these support vectors. Therefore, these points build an SVM classifier. For nonlinear data, the original coordinate area is converted into a separable space [35].

2.2.3. Decision Tree. Decision Tree is used to solve classification problems. It consists of root node, inner nodes, branches, and leaf nodes. It is organised in the form of a tree, where the root node represents the complete dataset, the internal nodes represent the features contained in the dataset, the branches represent the decision-making area, and the leaf nodes represent the outcome. Decisions are made on the basis of features selected in the dataset. When predicting dataset features, the algorithm starts from the root node. The algorithm compares the value of the root feature with the feature's values of the dataset, and in accordance with the comparison, it moves to the next nodes. The process continues to the next node, where the feature in the node is compared with the features in the next nodes, and the process continues until the leaf node is obtained.

2.2.4. Random Forest. Random Forest is used to solve classification problems. It works on the basis of ensemble learning, as it solves the problem by combining several classifiers to improve the performance of the algorithm.
The algorithm contains several classifiers of Decision Trees. Each Decision Tree works with a subset of data and average taken to improve prediction accuracy. Instead of taking prediction from one tree, the Random Forest algorithm takes prediction from each tree and works on prediction on the basis of majority voting.

2.2.5. Logistic Regression. Logistic Regression is one of the supervised machine learning algorithms used to solve classification problems to predict probability-based target variables. The target or dependent variables are binary variables that contain two classes; multinomial target variables have three or more unordered types or ordinal variables, where the target variable contains three or more ordered variables.

\section{Exploratory Data Analysis (EDA)}

This section focuses on data preprocessing, including missing data treatment, outlier removal, and feature correlation test. Figure 1 describes the structure applied to evaluate the performance of the algorithms on the two datasets for early diagnosis of heart disease. 
TABLE 2: Heart failure features with metrics from the Allied Hospital dataset.

\begin{tabular}{|c|c|c|c|}
\hline Features & Explanation & Range & Measurement \\
\hline Age & Age of patient in year & {$[40, \ldots, 95]$} & Year \\
\hline \multirow{2}{*}{ Anaemia } & $1=$ haematocrit levels lower than $36 \%$ & \multirow{2}{*}{0,1} & \multirow{2}{*}{ Boolean } \\
\hline & $0=$ haematocrit levels higher than $36 \%$ & & \\
\hline \multirow{2}{*}{ High blood pressure } & $1=$ patient has hypertension & \multirow{2}{*}{0,1} & \multirow{2}{*}{ Boolean } \\
\hline & $0=$ patient has no hypertension & & \\
\hline Creatinine phosphokinase & Level of CPK in blood & {$[23, \ldots, 7861]$} & $\mathrm{mcg} / \mathrm{L}$ \\
\hline \multirow{2}{*}{ Diabetes } & $1=$ patient has diabetes & \multirow{2}{*}{0,1} & \multirow{2}{*}{ Boolean } \\
\hline & $0=$ patient has no diabetes & & \\
\hline \multirow{2}{*}{ Sex } & $1=$ male & \multirow{2}{*}{0,1} & \multirow{2}{*}{ Boolean } \\
\hline & $0=$ female & & \\
\hline Platelets & Blood platelets & {$[25.01, \ldots, 850.00]$} & Kiloplatelets/mL \\
\hline Serum creatinine & Level of creatinine in blood & $\mathrm{mg} / \mathrm{dL}$ & {$[0.50, \ldots, 9.40]$} \\
\hline Serum sodium & Level of sodium in blood & $\mathrm{mEq} / \mathrm{L}$ & {$[114, \ldots, 148]$} \\
\hline \multirow{2}{*}{ Smoking } & $1=$ patient smokes & \multirow{2}{*}{0,1} & \multirow{2}{*}{ Boolean } \\
\hline & $0=$ patient does not smoke & & \\
\hline Time & Periodic follow-up of patient & Days & {$[4, \ldots, 285]$} \\
\hline \multirow{2}{*}{ Death event (target) } & 1 = patient died during follow-up & \multirow{2}{*}{0,1} & \multirow{2}{*}{ Boolean } \\
\hline & $0=$ patient did not die during follow-up & & \\
\hline
\end{tabular}

$\mathrm{mcg} / \mathrm{L}$ refers to micrograms per litre. $\mathrm{mL}$ refers to microlitre. $\mathrm{mEq} / \mathrm{L}$ refers to milliequivalents per litre.

TABle 3: Statistical operations for the Cleveland dataset.

\begin{tabular}{|c|c|c|c|c|c|c|c|c|c|c|c|c|c|c|}
\hline Statistical & Age & Sex & $\mathrm{cp}$ & trestbps & chol & fbs & restecg & thalach & exang & Oldpeak & Slope & $\mathrm{ca}$ & thal & Target \\
\hline Count & 303 & 303 & 303 & 303 & 303 & 303 & 303 & 303 & 303 & 303 & 303 & 303 & 303 & 303 \\
\hline Mean & 54.37 & 0.68 & 0.97 & 131.6 & 246.3 & 0.15 & 0.53 & 149.7 & 0.33 & 1.04 & 1.4 & 0.73 & 2.31 & 0.54 \\
\hline std & 9.08 & 0.47 & 1.03 & 17.54 & 51.83 & 0.36 & 0.53 & 22.91 & 0.47 & 1.16 & 0.62 & 1.02 & 0.61 & 0.5 \\
\hline Min & 29 & 0 & 0 & 94 & 126 & 0 & 0 & 71 & 0 & 0 & 0 & 0 & 0 & 0 \\
\hline Max & 77 & 1 & 3 & 200 & 564 & 1 & 2 & 202 & 1 & 6.2 & 2 & 4 & 3 & 1 \\
\hline
\end{tabular}

3.1. Description of Datasets. Two datasets of heart disease and failure data were collected from the UCI machine learning repository. The first dataset is called Cleveland (https://archive.ics.uci.edu/ml/datasets/heart+Disease) [36], which is commonly used by heart-disease diagnostic machine learning researchers. The Cleveland dataset consists of 303 records, with 76 features. However, the UCI repository provides approved 14 features that are most influential in the field of diagnosing heart disease. Table 1 describes the features, measures, and their ranges. Thirteen features could be used for diagnosing heart disease, and one target feature could be used to describe whether or not a disease exists.

The second dataset for predicting HF contains medical records of 299 patients with $\mathrm{HF}$ (https://archive.ics.uci.edu/ $\mathrm{ml} /$ machine-learning-databases/00519/) [37]. The dataset was collected from the Faisalabad Institute of Heart Disease and the Allied Hospital in Faisalabad. Table 2 describes the features, measurement, and range of HF prediction. Twelve features could predict HF in addition to the target feature that describes whether or not the patient died during follow-up. Table 2 also explains each feature and the subsections that represent each feature.
3.2. Statistical Feature Correlation Using Heat Map. A heat map is a graphical representation that shows the correlation between features and the percentage of correlation of each feature with the other. It also describes the correlation of all features with the target feature. Statistics is a set of computational tools used to interpret raw data and convert them into information to be understood. It is one of the tools used in the field of machine learning. Statistics and machine learning are two closely related fields. In this study, descriptive statistics were calculated on the dataset of heart disease (Cleveland dataset) and HF to obtain the features of common and correlated data samples as mean, standard deviation, and max and min values. Table 3 describes the statistical processes applied to the Cleveland dataset, where count refers to the number of features of dataset, mean refers to the mean between the features of the dataset, std refers to the standard deviation between the features of the dataset, and min and max refer to the minimum and maximum values amongst the features of the dataset. Descriptive statistics have a positive effect on graphic visualisations to easily understand raw data and relate the data to one another. Figure 2 illustrates the correlation between the features of the dataset with one another. "cp" (chest pain), 


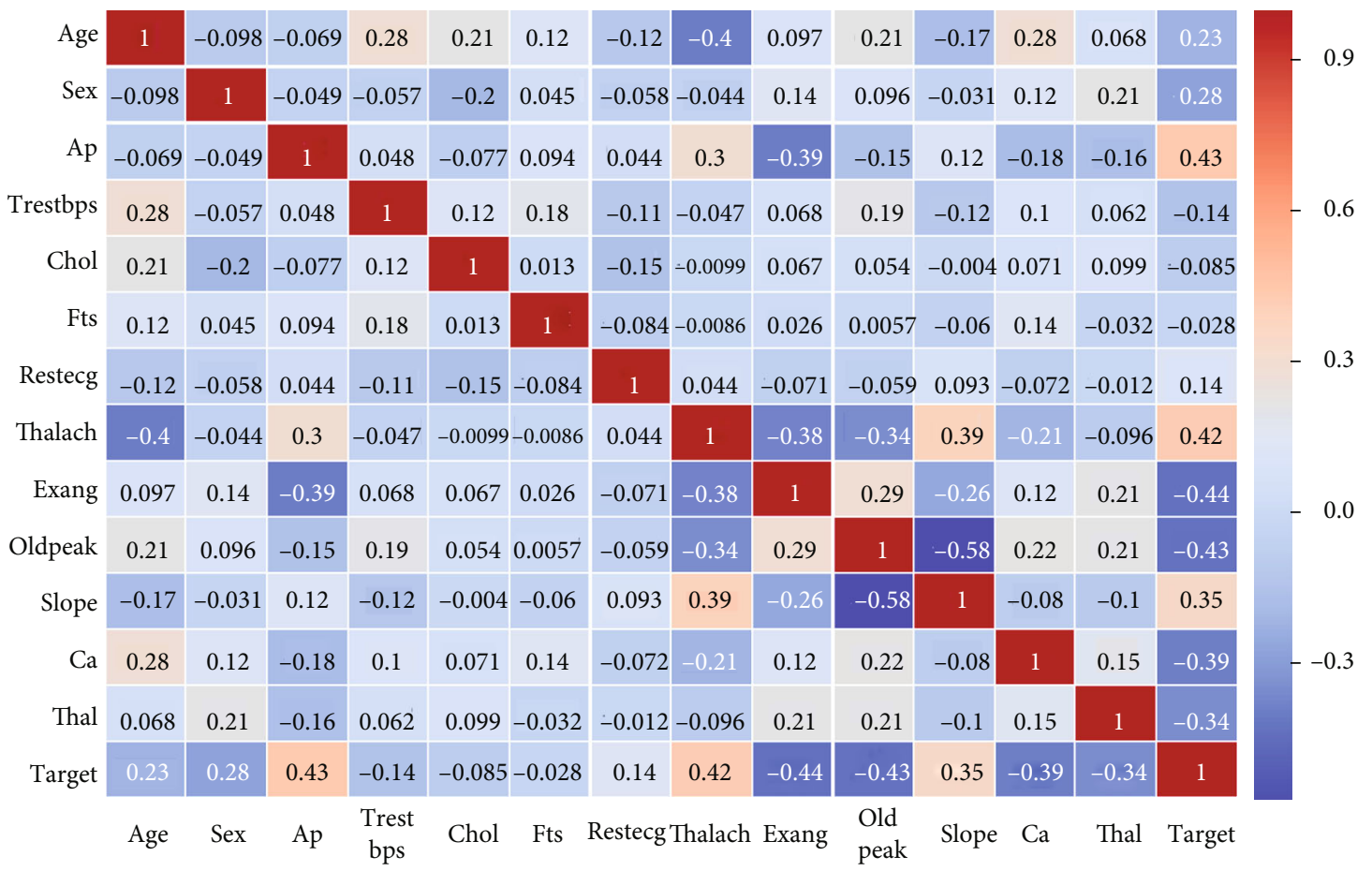

FIgUre 2: Feature correlation of the Cleveland dataset by using heat map.

"thalach," and "slope" features were correlated closely with the target feature, and the correlation of each distinctive with the other was noted.

3.3. Treatment of Missing Values. Datasets that contain missing values need to be addressed and cleaned up. Missing values result from patients missing out on some metrics when they undergo a test. The Cleveland dataset contained missing values, and the second dataset contained six missing values. Thus, statistical measures must be applied to replace the missing values. Statistical measures, such as mean, median, and standard deviation, are applied to replace numerical values. The mode method is also applied to replace nominal values. Table 4 describes the missing features for the Cleveland dataset after processing. The mean method was applied to replace the numerical values by calculating the mean for the features and replacing the missing value. The mode method was also applied to replace the missing nominal values by replacing the nominal value with the most common value in the features.

3.4. Balancing a Dataset. With regard to data balancing, the Cleveland dataset contains 165 people with heart disease and 138 people without heart disease. Thus, the dataset is balanced. Regarding the second dataset, the number of people who died during follow-up was 203, while 96 people did not die during follow-up; therefore, the dataset is unbalanced. To obtain satisfactory results, the dataset must be balanced during the training phase. In this study, the synthetic minority oversampling technique (SMOTE) was applied, which is one of the appropriate methods for balancing the dataset. SMOTE technique searches for minority classes and finds the nearest neighbor for each point (value) in the
TABle 4: Missing values.

\begin{tabular}{lc}
\hline Features & Missing values \\
\hline Age & 0 \\
Sex & 0 \\
cp & 0 \\
trestbps & 0 \\
chol & 0 \\
fbs & 0 \\
restecg & 0 \\
thalach & 0 \\
exang & 0 \\
Oldpeak & 0 \\
Slope & 0 \\
ca & 0 \\
thal & 0 \\
Target & 0 \\
dtype: int64 & 0 \\
\hline
\end{tabular}

minority class to generate new synthetic samples at a given point randomly, the mechanism continues until the dataset is balanced during the training phase, and the minority class becomes approximately equal to the majority class. Table 5 describes the second dataset before and after the application of the SMOTE technique, where it is noted that the cases of the minority class (die during follow-up) increased from 79 cases to 160 cases; thus, the two classes became equal during the training phase.

3.5. Data Conversion. Data processing is the ability to transform data into useful data that could be manipulated and 
TABLE 5: Balancing the dataset by SMOTE.

\begin{tabular}{lcccc}
\hline & \multicolumn{2}{c}{ Dataset } & \multicolumn{2}{c}{ Testing 20\% } \\
Phase & \multicolumn{2}{c}{ Training $80 \%$} & & \multicolumn{2}{c}{. } \\
Classes & Did not die during follow-up & Die during follow-up & Did not die during follow-up & Die during follow-up \\
\hline Before Oversampling & 160 & 79 & 43 & 17 \\
After Oversampling & 160 & 160 & 43 & 17 \\
\hline
\end{tabular}

TABle 6: Converting categorical data to dummy.

\begin{tabular}{|c|c|c|c|c|c|c|c|c|c|c|c|c|c|c|c|}
\hline$\overline{\text { Age }}$ & trestbps & chol & thalach & Oldpeak & Target & Sex_0 & Sex_1 & cp_0 & $\mathrm{cp} \_1$ & $\ldots$ & Slope_2 & ca_0 & ca_1 & ca_2 & ca_3 \\
\hline 0 & 63 & 145 & 233 & 150 & 2.3 & 1 & 0 & 1 & 0 & 0 & $\ldots$ & 0 & 1 & 0 & 0 \\
\hline 1 & 37 & 130 & 250 & 187 & 3.5 & 1 & 0 & 1 & 0 & 0 & $\ldots$ & 0 & 1 & 0 & 0 \\
\hline 2 & 41 & 130 & 204 & 172 & 1.4 & 1 & 1 & 0 & 0 & 1 & $\ldots$ & 1 & 1 & 0 & 0 \\
\hline 3 & 56 & 120 & 236 & 178 & 0.8 & 1 & 0 & 1 & 0 & 1 & $\ldots$ & 1 & 1 & 0 & 0 \\
\hline 4 & 57 & 120 & 354 & 163 & 0.6 & 1 & 1 & 0 & 1 & 0 & $\ldots$ & 1 & 1 & 0 & 0 \\
\hline
\end{tabular}

Table 7: Preprocessing of data by using standardization method.

\begin{tabular}{cccccc}
\hline & Age & trestbps & chol & thalach & Oldpeak \\
\hline 0 & 0.95 & 0.76 & -0.26 & 0.02 & 1.09 \\
1 & -1.92 & -0.09 & 0.07 & 1.63 & 2.12 \\
2 & -1.47 & -0.09 & -0.82 & 0.98 & 0.31 \\
3 & 0.18 & -0.66 & -0.2 & 1.24 & -0.21 \\
4 & 0.29 & -0.66 & 2.08 & 0.58 & -0.38 \\
\hline
\end{tabular}

analysed. In this study, categorical variables were converted into dummy variables, which include the values 0 and 1 . Dummy variables are useful for multiple groups in single regression equations. Table 6 describes the dataset after converting the categorical features to dummy.

3.6. Data Standardization. Preprocessing is one of the most important stages of data mining, and it leads to diagnostic accuracy in the following stages. In this study, data standardization method was applied to the two datasets. Standardization coordinates the data internally, ensures that all data have the same formatting and content, and gives the dataset more meaning. It transforms the dataset, in which its distribution has a mean of 0 and a standard deviation value of 1 . In this study, the standardization method was applied in accordance with Equation (1) and the dataset obtained its data distribution. Each feature in the dataset obtained its value subtracted from the mean and divided by the standard deviation of the whole dataset. Table 7 describes the application of the standardization method to four features of the dataset, namely, "trestbps," "chol," "thalach," and "oldpeak."

$$
z=\frac{x-\mu}{\sigma}
$$

where $x$ denotes the value of each feature, $\mu$ refers to the mean for each feature, $\sigma$ denotes the standard deviation of the dataset, and $z$ refers to the features in a standardised form.

\section{Feature Processing}

4.1. Feature Engineering. Feature engineering, also called feature creation, is the process of creating new features from the existing dataset for the purpose of training machine learning models and obtaining more reliable results. Usually, the feature engineering process is manual, relying on intuition, field knowledge, and data manipulation. It is also very tedious and limited. Thus, automated feature engineering helps data scientists create new features that are well correlated and use them for training. Table 8 describes the additional relevant extracted features correlated between two features, in which 60 features from the original Cleveland dataset containing 13 features were obtained. With these new features, the solution to the classification problem could be enhanced. The better the features, the better the results.

4.2. Feature Selection. Feature selection methods are aimed at reducing unimportant features and focusing on features that contribute to the most predictable feature of the target feature. Reducing the number of features reduces the computational cost of modelling and improves the performance of the model. The methods for selecting features by means of statistics include assessing the relationship between each feature and the target feature and selecting the input features that have the strongest correlation with the target feature. In this study, SelectKBest with the chi-square method was used to extract the best features from the dataset. The SelectKBest function uses this method as a score function to determine a score and the correlation between each feature and target feature. It passes chi-square to determine the score between each feature and the target feature. If the resulting value is lower, then the feature is independent of the target feature, while higher resulting value indicates that the feature is not randomly related to the target feature. Table 8 describes how the SelectKBest function automatically returns the first $K$ features with the highest scores of the Cleveland dataset. The exang_oldpeak2 feature, which is correlated between exang feature and oldpeak 2 feature, 
TABLE 8: Creation of features and arranging the best features.

\begin{tabular}{|c|c|c|}
\hline No & Feature & Score \\
\hline 1 & exang_oldpeak2 & 652.854396 \\
\hline 2 & exang_ca & 263.212119 \\
\hline 3 & Sex_oldpeak2 & 252.657949 \\
\hline 4 & thal_oldpeak2 & 247.914913 \\
\hline 5 & exang_trestbps2 & 241.749732 \\
\hline 6 & Thalach & 186.180286 \\
\hline 7 & Oldpeak2 & 171.4864 \\
\hline 8 & fbs_oldpeak2 & 164.89897 \\
\hline 9 & Age2_oldpeak2 & 139.151372 \\
\hline 10 & exang_chol2 & 131.365522 \\
\hline 11 & thal_trestbps2 & 116.88462 \\
\hline 12 & thal_chol2 & 113.985724 \\
\hline 13 & thal_ca & 90.668503 \\
\hline 14 & Sex_trestbps2 & 78.162433 \\
\hline 15 & Sex_ca & 77.302537 \\
\hline 16 & Oldpeak & 71.692782 \\
\hline 17 & $\mathrm{Ca}$ & 71.020719 \\
\hline 18 & $\mathrm{Cp}$ & 62.116086 \\
\hline 19 & Age2_ca & 54.956199 \\
\hline 20 & Age2_trestbps2 & 53.221349 \\
\hline 21 & restecg_cp & 51.837075 \\
\hline 22 & fbs_ca & 43.441045 \\
\hline 23 & exang & 38.518849 \\
\hline 24 & Age2_chol2 & 36.438097 \\
\hline 25 & Sex_chol2 & 35.823916 \\
\hline 26 & fbs_cp & 32.072291 \\
\hline 27 & restecg_thalach2 & 29.718076 \\
\hline 28 & exang_thalach2 & 27.279766 \\
\hline 29 & Age & 22.210517 \\
\hline 30 & Chol & 21.690747 \\
\hline 31 & exang_slope & 20.48139 \\
\hline 32 & exang_cp & 18.443334 \\
\hline 33 & restecg_slope & 18.246965 \\
\hline 34 & trestbps & 15.094591 \\
\hline 35 & restecg_trestbps2 & 12.462827 \\
\hline 36 & thal_thalach2 & 12.403249 \\
\hline 37 & Slope & 9.677715 \\
\hline 38 & restecg_oldpeak2 & 8.249627 \\
\hline 39 & Sex & 7.72169 \\
\hline 40 & thal_slope & 7.199342 \\
\hline 41 & Sex_thalach2 & 5.89906 \\
\hline 42 & fbs_trestbps2 & 5.897746 \\
\hline 43 & thal_cp & 5.838268 \\
\hline 44 & Thal & 5.75303 \\
\hline 45 & thalach2 & 5.688919 \\
\hline 46 & restecg_chol2 & 5.639162 \\
\hline 47 & fbs_slope & 5.480661 \\
\hline 48 & Age2_thalach2 & 5.466855 \\
\hline
\end{tabular}

Table 8: Continued.

\begin{tabular}{lcc}
\hline No & Feature & Score \\
\hline 49 & fbs_thalach2 & 4.64652 \\
50 & Age2_slope & 4.031292 \\
51 & Sex_slope & 2.987813 \\
52 & restecg & 2.877743 \\
53 & Age2_cp & 2.324974 \\
54 & fbs_chol2 & 2.116344 \\
55 & Age2 & 2.00334 \\
56 & trestbps2 & 1.655964 \\
57 & chol2 & 0.877493 \\
58 & Sex_cp & 0.564425 \\
59 & restecg_ca & 0.256374 \\
60 & fbs & 0.184946 \\
\hline
\end{tabular}

had the highest score of 652.85 , while the lowest fbs feature score was 0.18 .

\section{Experimental Result and Discussion}

5.1. Splitting Datasets. The Cleveland dataset consisted of 303 records for two classes: heart disease (class 1), which contained 165 records by $54.46 \%$, and normal (class 0 ), which contained 138 records by $45.54 \%$. The second dataset (HF) contains 299 records for two classes: died during follow-up (class 1), containing 203 records by $67.87 \%$, and did not die during follow-up (class 0 ) containing 96 records by $32.13 \%$. After balancing the second dataset, the two classes became equal to 160 cases during the training phase. Table 9 describes the distribution of the two datasets for the two groups during the training and testing phases.

5.2. Evaluation Criteria. Four qualitative measures were used, namely, accuracy, precision, recall, and F1score, to evaluate the proposed systems on the two datasets, as shown in Equations (2)-(5).

$$
\begin{aligned}
\text { Accuracy } & =\frac{\mathrm{TN}+\mathrm{TP}}{\mathrm{TN}+\mathrm{TP}+\mathrm{FN}+\mathrm{FP}} * 100 \%, \\
\text { Precision } & =\frac{\mathrm{TP}}{\mathrm{TP}+\mathrm{FP}} * 100 \%, \\
\text { Recall } & =\frac{\mathrm{TP}}{\mathrm{TP}+\mathrm{FN}} * 100 \%, \\
\mathrm{~F} 1 \text { score } & =2 * \frac{\text { Precision } * \text { recall }}{\text { Precision }+ \text { recall }} * 100,
\end{aligned}
$$

where TP is the number of heart disease samples that are correctly classified, TN is the number of nonheart disease samples that are correctly classified, FN is the number of heart disease samples classified as nonheart disease, and FP is the number of nonheart disease samples classified as heart disease [38].

5.3. Results for the Cleveland Dataset. Several machine learning algorithms have been applied to predict heart disease for 
TABLE 9: Splitting the datasets.

\begin{tabular}{lcccc}
\hline $\begin{array}{l}\text { Dataset } \\
\text { Class }\end{array}$ & Cleveland dataset & & \multicolumn{2}{c}{ HF dataset } \\
Heart disease & Normal & Died during follow-up & Did not die during follow-up \\
\hline Training & 133 & 109 & 160 & 160 \\
Testing & 32 & 29 & 43 & 17 \\
\hline
\end{tabular}

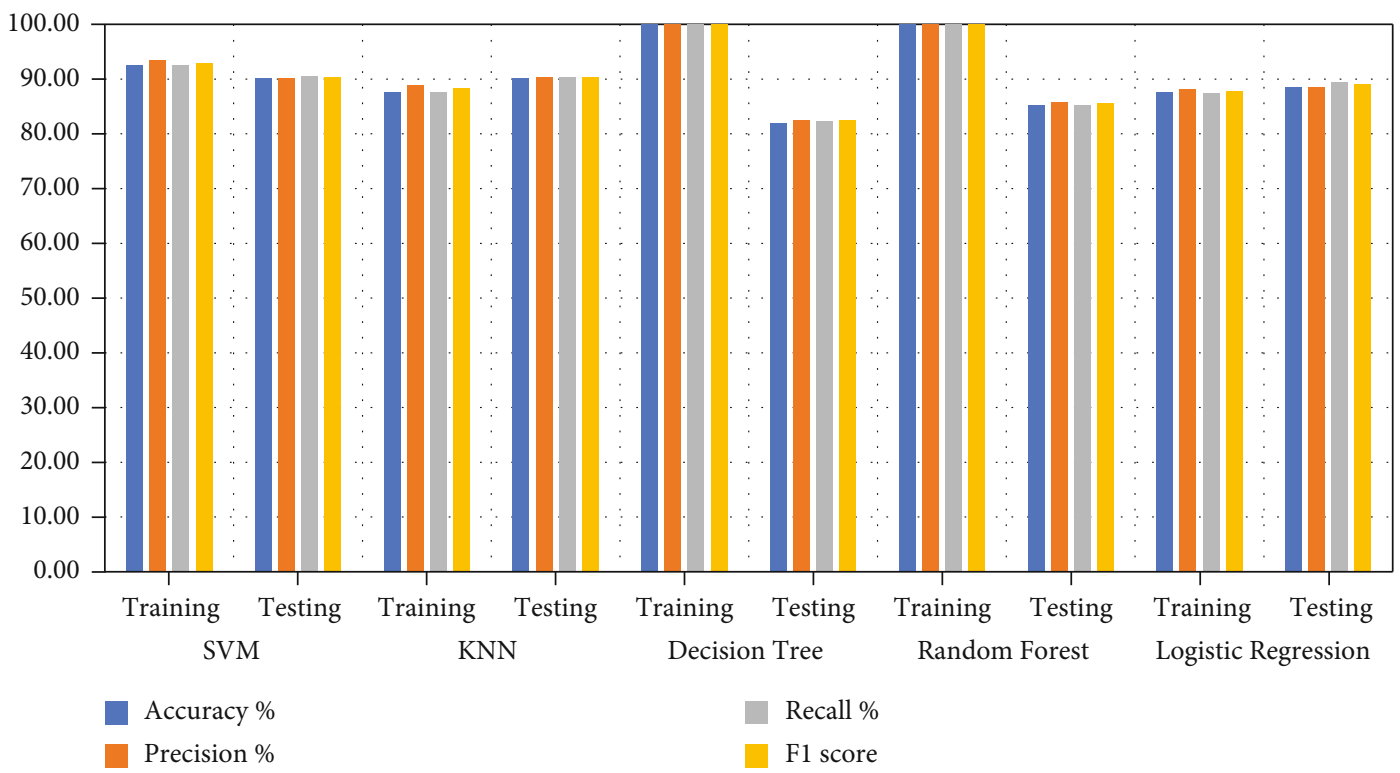

FIGURE 3: Evaluating the performance of five classifiers on the Cleveland dataset.

TABLE 10: Results of diagnosing heart disease (Cleveland dataset) by using five machine learning algorithms.

\begin{tabular}{|c|c|c|c|c|c|c|c|c|c|c|}
\hline \multirow{2}{*}{$\begin{array}{l}\text { Classifiers } \\
\text { Criteria }\end{array}$} & \multicolumn{2}{|c|}{ SVM } & \multicolumn{2}{|c|}{$\mathrm{KNN}$} & \multicolumn{2}{|c|}{ Decision Tree } & \multicolumn{2}{|c|}{ Random Forest } & \multicolumn{2}{|c|}{ Logistic Regression } \\
\hline & $\begin{array}{c}\text { Training } \\
80 \%\end{array}$ & $\begin{array}{c}\text { Testing } \\
20 \%\end{array}$ & $\begin{array}{c}\text { Training } \\
80 \%\end{array}$ & $\begin{array}{c}\text { Testing } \\
20 \%\end{array}$ & $\begin{array}{c}\text { Training } \\
80 \%\end{array}$ & $\begin{array}{c}\text { Testing } \\
20 \%\end{array}$ & $\begin{array}{c}\text { Training } \\
80 \%\end{array}$ & $\begin{array}{c}\text { Testing } \\
20 \%\end{array}$ & $\begin{array}{c}\text { Training } \\
80 \%\end{array}$ & $\begin{array}{c}\text { Testing } \\
20 \%\end{array}$ \\
\hline $\begin{array}{l}\text { Accuracy } \\
(\%)\end{array}$ & 92.56 & 90.16 & 87.60 & 90.16 & 100 & 81.97 & 100 & 85.25 & 87.60 & 88.52 \\
\hline $\begin{array}{l}\text { Precision } \\
(\%)\end{array}$ & 93.45 & 90.12 & 88.82 & 90.26 & 100 & 82.43 & 100 & 85.82 & 88.19 & 88.56 \\
\hline Recall (\%) & 92.52 & 90.45 & 87.63 & 90.38 & 100 & 82.39 & 100 & 85.29 & 87.44 & 89.46 \\
\hline F1 score & 92.98 & 90.28 & 88.23 & 90.32 & 100 & 82.41 & 100 & 85.55 & 87.81 & 89.00 \\
\hline
\end{tabular}

patient survival. Classification and hyperparameter algorithms that produce optimal networks have been optimised to reduce function loss and to obtain high diagnostic performance. Tuning hyperparameter is an important process for determining the behaviour of machine learning networks during training. In this study, machine learning models were applied to the dataset containing 13 original features of 303 patients. New features were created through the correlation of the original features, and they were expanded to 60 features for each patient. The dataset was divided randomly into $80 \%$ for training (242 patients) and 20\% for testing (61 patients). Figure 3 shows the performance of classification algorithms on the dataset during the training and testing phases. Table 10 shows the diagnostic results for heart disease by using five machine learning algorithms during the training and testing processes. During the training phase, Decision Tree and Random Forest obtained the best results of $100 \%$ for all measures. However, during the testing phase, SVM and KNN algorithms achieved the best results, with an approximate rate of $90 \%$ for all measures. Logistic Regression obtained the lowest result during the training and testing phases amongst all the algorithms. During the training phase, SVM, KNN, Decision Tree, Random Forest, and Logistic Regression reached accuracy scores of $92.56 \%$, $87.60 \%, 100 \%, 100 \%$, and $87.60 \%$, respectively; in the testing phase, their accuracy scores were $90.16 \%, 90.16 \%, 81.97 \%$, $85.25 \%$, and $88.52 \%$, respectively. For the precision, during the training phase, SVM, KNN, Decision Tree, Random 
TABLE 11: Results of diagnosing heart disease as the category using five machine learning algorithms.

\begin{tabular}{|c|c|c|c|c|c|c|c|}
\hline $\mathrm{SN}$ & Classifiers & Division of data & Class & Precision (\%) & Recall (\%) & F1 score (\%) & Number of patients \\
\hline 1 & \multirow{4}{*}{ SVM } & \multirow{2}{*}{ Training (80\%) } & 0 & 93 & 90 & 92 & 109 \\
\hline 2 & & & 1 & 92 & 95 & 93 & 133 \\
\hline 3 & & \multirow{2}{*}{ Testing (20\%) } & 0 & 87 & 93 & 90 & 29 \\
\hline 4 & & & 1 & 93 & 88 & 90 & 32 \\
\hline 5 & \multirow{4}{*}{$\mathrm{KNN}$} & \multirow{2}{*}{ Training (80\%) } & 0 & 88 & 84 & 86 & 109 \\
\hline 6 & & & 1 & 88 & 90 & 89 & 133 \\
\hline 7 & & \multirow{2}{*}{ Testing (20\%) } & 0 & 90 & 90 & 90 & 29 \\
\hline 8 & & & 1 & 91 & 91 & 91 & 32 \\
\hline 9 & \multirow{4}{*}{ Decision Tree } & \multirow{2}{*}{ Training (80\%) } & 0 & 100 & 100 & 100 & 109 \\
\hline 10 & & & 1 & 100 & 100 & 100 & 133 \\
\hline 11 & & \multirow{2}{*}{ Testing (20\%) } & 0 & 78 & 86 & 82 & 29 \\
\hline 12 & & & 1 & 86 & 78 & 82 & 32 \\
\hline 13 & \multirow{4}{*}{ Random Forest } & \multirow{2}{*}{ Training (80\%) } & 0 & 100 & 100 & 100 & 109 \\
\hline 14 & & & 1 & 100 & 100 & 100 & 133 \\
\hline 15 & & \multirow{2}{*}{ Testing (20\%) } & 0 & 83 & 86 & 85 & 29 \\
\hline 16 & & & 1 & 87 & 84 & 86 & 32 \\
\hline 17 & \multirow{4}{*}{ Logistic Regression } & \multirow{2}{*}{ Training (80\%) } & 0 & 88 & 83 & 86 & 109 \\
\hline 18 & & & 1 & 87 & 91 & 89 & 133 \\
\hline 19 & & \multirow{2}{*}{ Testing (20\%) } & 0 & 87 & 90 & 88 & 29 \\
\hline 20 & & & 1 & 90 & 88 & 89 & 32 \\
\hline
\end{tabular}

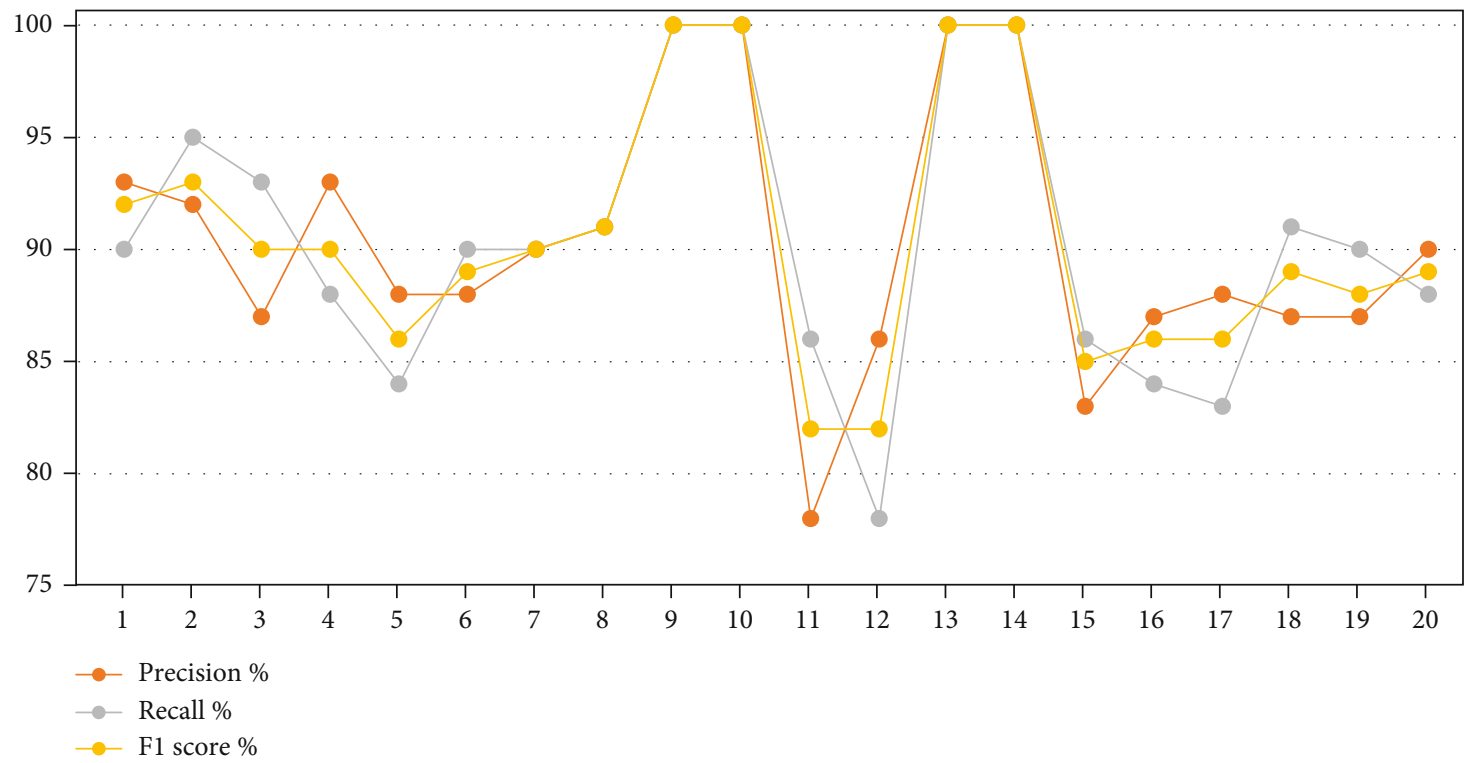

FIgURE 4: The performance of classification algorithms for each class.

Forest, and Logistic Regression reached of 93.45\%, $88.82 \%$, $100 \%, 100 \%$, and $88.19 \%$, respectively; in the testing phase, their precision rates were $90.12 \%, 90.26 \%, 82.43 \%, 85.82 \%$, and $88.56 \%$, respectively. During the training phase, SVM, KNN, Decision Tree, Random Forest, and Logistic Regression reached recall rates of $92.52 \%, 87.63 \%, 100 \%, 100 \%$, and $87.44 \%$, respectively; in the testing phase, their recall rates were $90.45 \%, 90.38 \%, 82.39 \%, 85.29 \%$, and $89.46 \%$, respectively. For the F1 score, during the training phase, SVM, KNN, Decision Tree, Random Forest, and Logistic Regression reached 92.98\%, 88.23\%, 100\%, $100 \%$, and $87.81 \%$, respectively; In the testing phase, their F1 scores were 90.28\%, 90.32\%, 82.41\%, 85.55\%, and $89 \%$, respectively.

Table 11 and Figure 4 describe the analysis of the results obtained in depth at each class, where heart disease $=1$ and nonheart disease $=0$. The dataset was divided into $80 \%$ for training and $20 \%$ for testing. The training data were further 


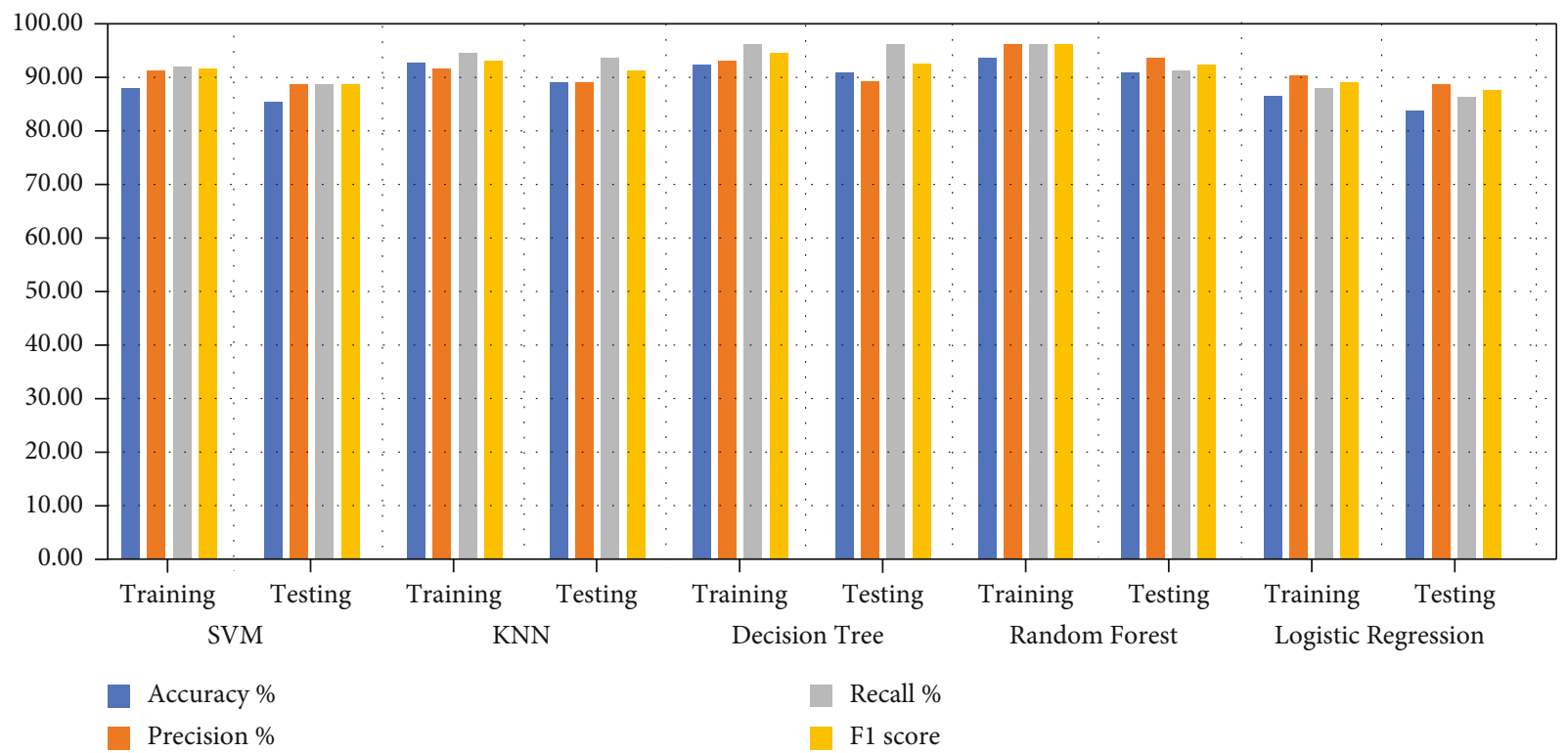

Figure 5: Evaluating the performance of five classifiers on the heart failure dataset.

TABle 12: Prediction results of heart failure by using five machine learning algorithms.

\begin{tabular}{|c|c|c|c|c|c|c|c|c|c|c|}
\hline \multirow{2}{*}{$\begin{array}{l}\text { Classifiers } \\
\text { Criteria }\end{array}$} & \multicolumn{2}{|c|}{ SVM } & \multicolumn{2}{|c|}{ KNN } & \multicolumn{2}{|c|}{ Decision Tree } & \multicolumn{2}{|c|}{ Random Forest } & \multicolumn{2}{|c|}{ Logistic Regression } \\
\hline & $\begin{array}{c}\text { Training } \\
80 \%\end{array}$ & $\begin{array}{c}\text { Testing } \\
20 \%\end{array}$ & $\begin{array}{c}\text { Training } \\
80 \%\end{array}$ & $\begin{array}{c}\text { Testing } \\
20 \%\end{array}$ & $\begin{array}{c}\text { Training } \\
80 \%\end{array}$ & $\begin{array}{c}\text { Testing } \\
20 \%\end{array}$ & $\begin{array}{c}\text { Training } \\
80 \%\end{array}$ & $\begin{array}{c}\text { Testing } \\
20 \%\end{array}$ & $\begin{array}{c}\text { Training } \\
80 \%\end{array}$ & $\begin{array}{c}\text { Testing } \\
20 \%\end{array}$ \\
\hline $\begin{array}{l}\text { Accuracy } \\
(\%)\end{array}$ & 92.35 & 90.00 & 96.82 & 93.33 & 96.46 & 95.00 & 97.68 & 95.00 & 91.05 & 88.33 \\
\hline $\begin{array}{l}\text { Precision } \\
(\%)\end{array}$ & 95.41 & 93.02 & 95.76 & 93.33 & 97.11 & 93.48 & 100.00 & 97.62 & 94.52 & 93.00 \\
\hline Recall (\%) & 96.10 & 93.02 & 98.51 & 97.67 & 100.00 & 100.00 & 100.00 & 95.35 & 92.39 & 90.90 \\
\hline F1 score & 95.75 & 93.02 & 97.12 & 95.45 & 98.53 & 96.63 & 100.00 & 96.47 & 93.44 & 91.93 \\
\hline
\end{tabular}

divided into 133 for heart disease and 109 for nonheart disease, while the test data were divided into 32 for heart disease and 29 for nonheart disease. During the training phase, Decision Tree and Random Forest achieved the best results for diagnosing heart and nonheart diseases by $100 \%$ for all measures. However, during the testing phase, KNN achieved better than the rest of the algorithms, with rates of $90 \%$ for all criteria when diagnosing negative cases (nonheart disease) and 91\% for all criteria when diagnosing positive cases (heart disease). First, in the analysis and interpretation of the results of the diagnosis of heart disease (class 1) during the training phase, SVM, KNN, Decision Tree, Random Forest, and Logistic Regression reached a precision of $92 \%, 88 \%$, $100 \%, 100 \%$, and $87 \%$, respectively; in the testing phase, their precision was $93 \%, 91 \%, 86 \%, 87 \%$, and $90 \%$, respectively. For the recall, during the training phase, SVM, KNN, Decision Tree, Random Forest, and Logistic Regression reached a recall of $95 \%, 90 \%, 100 \%, 100 \%$, and $91 \%$, respectively; in the testing phase, their recall was $88 \%$, $91 \%, 78 \%, 84 \%$, and $88 \%$, respectively, while for the F1 score during the training phase, SVM, KNN, Decision Tree, Random Forest, and Logistic Regression reached a recall of $93 \%, 89 \%, 100 \%, 100 \%$, and $89 \%$, respectively; in the testing phase, their F1 scores were 90\%, 91\%, 82\%, 86\%, and 89\%, respectively. Second, in the analysis and interpretation of the results of the diagnosis of nonheart disease (class 0 ) during the training phase, SVM, KNN, Decision Tree, Random Forest, and Logistic Regression reached a precision of 93\%, $88 \%, 100 \%, 100 \%$, and $88 \%$, respectively; in the testing phase, their precision was $87 \%, 90 \%, 78 \%, 83 \%$, and $87 \%$, respectively. For the recall, during the training phase, SVM, KNN, Decision Tree, Random Forest, and Logistic Regression reached a recall of $90 \%, 84 \%, 100 \%, 100 \%$, and $83 \%$, respectively; in the testing phase, their recall was $93 \%, 90 \%, 86 \%, 86 \%$, and $90 \%$, respectively, while for the F1 score during the training phase, SVM, KNN, Decision Tree, Random Forest, and Logistic Regression reached a recall of $92 \%, 86 \%, 100 \%, 100 \%$, and $86 \%$, respectively; in the testing phase, their F1 scores were 90\%, 90\%, 82\%, $85 \%$, and $88 \%$, respectively.

5.4. Results of HF Dataset. A medical dataset containing 299 patients with HF was analysed. This section describes the outcomes that predicted patient survival during the followup period. The features were arranged in accordance with the correlation with the target feature (death event), and 
TABLE 13: Result prediction of heart failure as the category using five machine learning algorithms.

\begin{tabular}{|c|c|c|c|c|c|c|c|}
\hline $\mathrm{SN}$ & Classifiers & Division of data & Class & Precision (\%) & Recall (\%) & F1 score (\%) & Number of patients \\
\hline 1 & \multirow{4}{*}{ SVM } & \multirow{2}{*}{ Training (80\%) } & 0 & 92 & 95 & 95 & 79 \\
\hline 2 & & & 1 & 93 & 96 & 97 & 160 \\
\hline 3 & & \multirow{2}{*}{ Testing (20\%) } & 0 & 92 & 91 & 92 & 17 \\
\hline 4 & & & 1 & 94 & 94 & 94 & 43 \\
\hline 5 & \multirow{4}{*}{$\mathrm{KNN}$} & \multirow{2}{*}{ Training (80\%) } & 0 & 89 & 97 & 96 & 79 \\
\hline 6 & & & 1 & 91 & 99 & 98 & 160 \\
\hline 7 & & \multirow{2}{*}{ Testing (20\%) } & 0 & 92 & 97 & 94 & 17 \\
\hline 8 & & & 1 & 94 & 98 & 96 & 43 \\
\hline 9 & \multirow{4}{*}{ Decision Tree } & \multirow{2}{*}{ Training (80\%) } & 0 & 97 & 100 & 98 & 79 \\
\hline 10 & & & 1 & 98 & 100 & 99 & 160 \\
\hline 11 & & \multirow{2}{*}{ Testing (20\%) } & 0 & 92 & 100 & 95 & 17 \\
\hline 12 & & & 1 & 94 & 100 & 97 & 43 \\
\hline 13 & \multirow{4}{*}{ Random Forest } & \multirow{2}{*}{ Training (80\%) } & 0 & 100 & 100 & 100 & 79 \\
\hline 14 & & & 1 & 100 & 100 & 100 & 160 \\
\hline 15 & & \multirow{2}{*}{ Testing (20\%) } & 0 & 98 & 96 & 97 & 17 \\
\hline 16 & & & 1 & 97 & 95 & 96 & 43 \\
\hline 17 & \multirow{4}{*}{ Logistic Regression } & \multirow{2}{*}{ Training (80\%) } & 0 & 94 & 93 & 94 & 79 \\
\hline 18 & & & 1 & 96 & 91 & 93 & 160 \\
\hline 19 & & \multirow{2}{*}{ Testing (20\%) } & 0 & 93 & 90 & 91 & 17 \\
\hline 20 & & & 1 & 93 & 92 & 93 & 43 \\
\hline
\end{tabular}

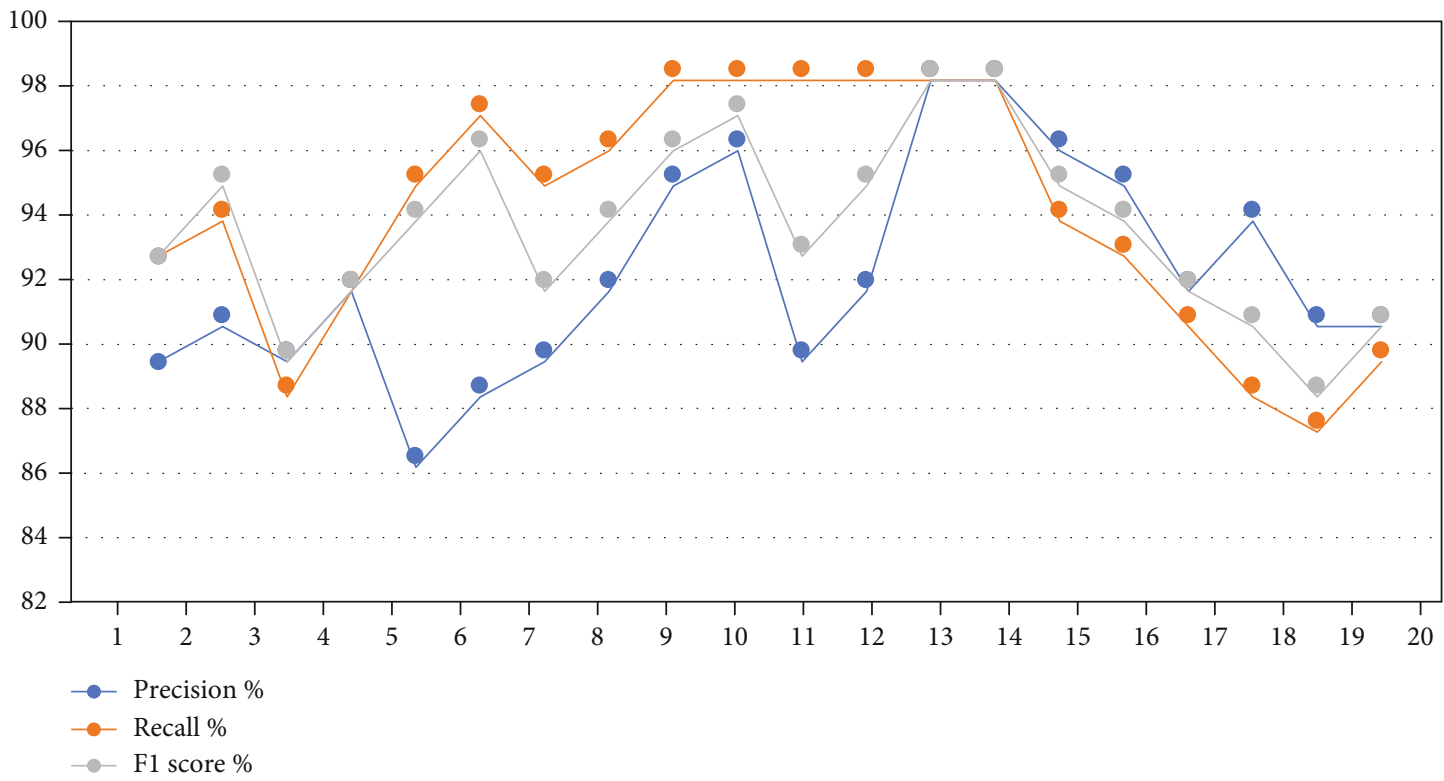

Figure 6: The performance of classification algorithms for each class.

the data lost due to the loss of some tests during patient examination were processed and replaced. Correlated features were created between two features, and they have an effective effect on increasing the accuracy of prediction. Hyperparameter classification algorithms were adjusted to reduce the loss function and obtain high predictive results. The dataset was divided into $80 \%$ for training (160 patients died during follow-up, and 79 patients did not die during follow-up) and $20 \%$ for testing (43 patients died during follow-up, and 17 patients did not die during follow-up). Figure 5 illustrates the evaluation of the dataset on the performance of the algorithms during the training and testing phases. Table 12 shows the results for predicting HF by using five classification algorithms during the training and testing phases. Random Forest achieved the best performance during both phases, followed by Decision Tree, 
TABLe 14: Accuracy of diagnosing two dataset using five machine learning algorithms.

\begin{tabular}{|c|c|c|c|c|c|c|c|c|c|c|}
\hline \multirow{2}{*}{$\begin{array}{l}\text { Dataset } \\
\text { Dataset }\end{array}$} & \multicolumn{2}{|c|}{ SVM } & \multicolumn{2}{|c|}{$\mathrm{KNN}$} & \multicolumn{2}{|c|}{ Decision Tree } & \multicolumn{2}{|c|}{ Random Forest } & \multicolumn{2}{|c|}{ Logistic Regression } \\
\hline & Training & Testing & Training & Testing & Training & Testing & Training & Testing & Training & Testing \\
\hline Cleveland & 92.56 & 90.16 & 87.60 & 90.16 & 100 & 81.97 & 100 & 85.25 & 87.60 & 88.52 \\
\hline $\mathrm{HF}$ & 92.35 & 90.00 & 96.82 & 93.33 & 96.46 & 95.00 & 97.68 & 95.00 & 91.05 & 88.33 \\
\hline
\end{tabular}

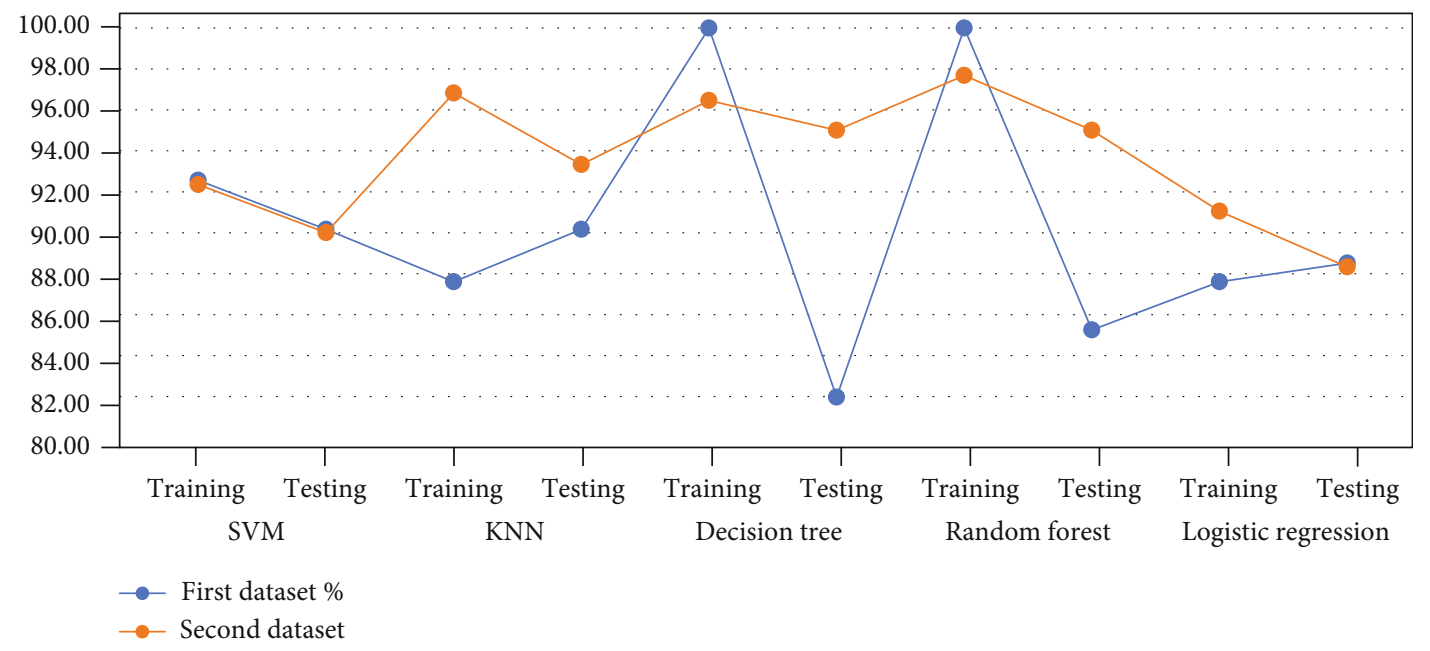

Figure 7: Comparison of system performance on the diagnostic accuracy in two datasets.

KNN, SVM, and Logistic Regression. During the training phase, SVM, KNN, Decision Tree, Random Forest, and Logistic Regression reached an accuracy of $92.35 \%, 96.82 \%$, $96.46 \%, 97.68 \%$, and $91.05 \%$, respectively; in the testing phase, their accuracy was 90\%, 93.33\%, 95\%, 95\%, and $88.33 \%$, respectively. For the precision, during the training phase, SVM, KNN, Decision Tree, Random Forest, and Logistic Regression reached of $95.41 \%, 95.76 \%, 97.11 \%$, $100 \%$, and $94.52 \%$, respectively; in the testing phase, their precision rates were 93.02\%, 93.33\%, 93.48\%, 97.62\%, and 93\%, respectively. During the training phase, SVM, KNN, Decision Tree, Random Forest, and Logistic Regression reached recall rates of $96.10 \%, 98.51 \%, 100 \%, 100 \%$, and $92.39 \%$, respectively; in the testing phase, their recall rates were $93.02 \%, 97.67 \%, 100 \%, 95.35 \%$, and $90 \%$, respectively. For the F1 score, during the training phase, SVM, KNN, Decision Tree, Random Forest, and Logistic Regression reached $95.75 \%, 97.12 \%, 98.53 \%, 100 \%$, and $93.44 \%$, respectively; in the testing phase, their F1 scores were $93.02 \%$, $95.45 \%, 96.63 \%, 96.47 \%$, and $91.93 \%$, respectively.

Table 13 and Figure 6 describe the HF results predicted using the five machine learning algorithms for each class ( 1 = died during follow-up and $0=$ did not die during follow-up). The training data were divided into 160 for class 1 and 79 for class 0 , while the test data were divided into 43 for class 1 and 17 for class 0. Random Forest achieved the best result during the training phase for both classes, with $100 \%$ for each criterion (precision, recall, and F1 score). During the testing phase, Random Forest also achieved the best precision for predicting HF, with $97 \%$ for class 1 and 98\% for class 0 . Meanwhile, Decision Tree achieved the best recall of $100 \%$ for both classes. Random Forest showed the best F1 score of $96 \%$ for predicting positive cases and $97 \%$ for predicting negative cases. First, in the analysis and interpretation of the results of the diagnosis of died during follow-up (class 1) during the training phase, SVM, KNN, Decision Tree, Random Forest, and Logistic Regression reached a precision of $93 \%, 91 \%, 98 \%, 100 \%$, and $96 \%$, respectively; in the testing phase, their precision was $94 \%$, $94 \%, 94 \%, 97 \%$, and $93 \%$, respectively. For the recall, during the training phase, SVM, KNN, Decision Tree, Random Forest, and Logistic Regression reached a recall of 96\%, 99\%, $100 \%, 100 \%$, and $91 \%$, respectively; in the testing phase, their recall was $94 \%, 98 \%, 100 \%, 95 \%$, and $92 \%$, respectively, while for the F1 score during the training phase, SVM, KNN, Decision Tree, Random Forest, and Logistic Regression reached a recall of $97 \%, 98 \%, 99 \%, 100 \%$, and 93\%, respectively; in the testing phase, their F1 scores were 94\%, 96\%, $97 \%, 96 \%$, and $93 \%$, respectively. Second, in the analysis and interpretation of the results of the diagnosis of did not die during follow-up (class 0) during the training phase, SVM, KNN, Decision Tree, Random Forest, and Logistic Regression reached a precision of $92 \%, 89 \%, 97 \%, 100 \%$, and $94 \%$, respectively; in the testing phase, their precision was $92 \%, 92 \%, 92 \%, 98 \%$, and $93 \%$, respectively. For the recall, during the training phase, SVM, KNN, Decision Tree, Random Forest, and Logistic Regression reached a recall of $95 \%, 97 \%, 100 \%, 100 \%$, and $93 \%$, respectively; in the testing phase, their recall was $91 \%, 97 \%, 100 \%, 96 \%$, and $90 \%$, respectively, while for the F1 score during the training phase, SVM, KNN, Decision Tree, Random Forest, and Logistic Regression reached a recall of 95\%, 96\%, 98\%, 100\%, and 
TABLE 15: Comparison of the performance between the proposed system and previous studies.

\begin{tabular}{|c|c|c|c|c|}
\hline Previous studies & Accuracy (\%) & Precision $(\%)$ & Recall (\%) & F1 score $(\%)$ \\
\hline Arabasadi et al. [14] & 93.85 & - & 97 & - \\
\hline Maji and Arora [15] & 77.4 & - & 77.4 & - \\
\hline Reddy et al. [39] & 90 & - & 91 & - \\
\hline Amin et al. [40] & 78.15 & 78.15 & - & 80.25 \\
\hline Feshki and Shijani [19] & 91.94 & 91.9 & 93 & - \\
\hline Pouriyeh et al. [41] & 77.55 & 77.4 & 83 & 80.1 \\
\hline Chicco and Jurman [42] & 83.8 & - & 72 & 71.9 \\
\hline Proposed model first dataset for training & 97.68 & 100 & 100 & 100 \\
\hline Proposed model second dataset for training & 100 & 100 & 100 & 100 \\
\hline Proposed model first dataset for testing & 90.16 & 90.26 & 90.38 & 90.32 \\
\hline Proposed model second dataset for testing & 95 & 97.62 & 95.35 & 96.47 \\
\hline
\end{tabular}

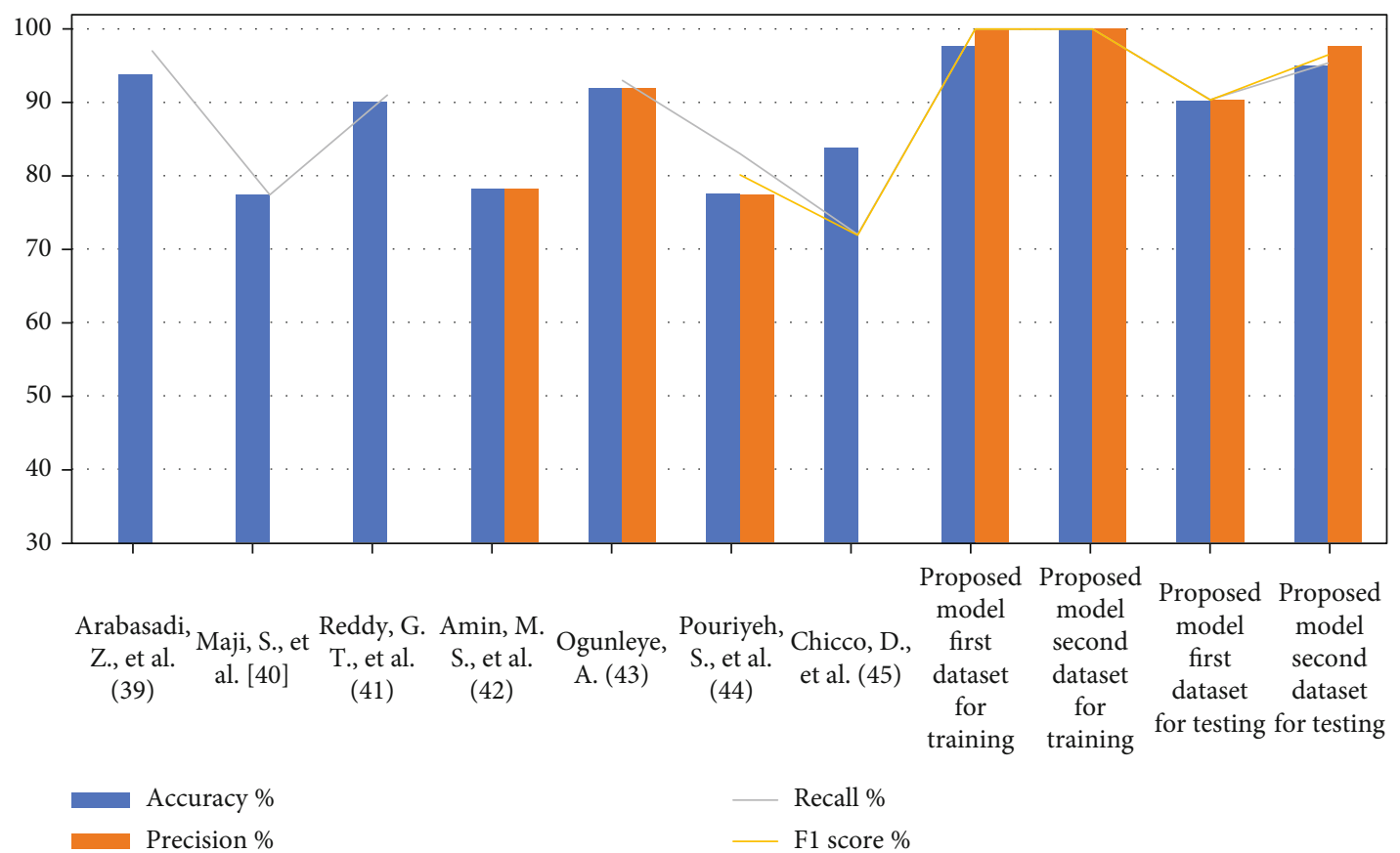

FIGURE 8: The performance of our systems with the previous studies.

94\%, respectively; in the testing phase, their F1 scores were $92 \%, 94 \%, 95 \%, 97 \%$, and $91 \%$, respectively.

5.5. Comparison of the Performance of Algorithms between the Two Datasets. Similar data processing methods, preprocessing, processing features and arranging them in order of importance, and classification algorithms, were applied to the two datasets, Cleveland and HF datasets. Through the analyses in the previous sections, the diagnostic systems were able to evaluate the HF dataset, with an accuracy that exceeded the evaluation of the Cleveland dataset during the test phase. Table 14 and Figure 7 describe the analytical results to compare the performance of machine learning algorithms on the two datasets. First, the performance of the SVM, KNN, Decision Tree, Random Forest, and Logistic Regression on the Cleveland dataset during the training phase reached to an accuracy of $92.56 \%, 87.60 \%, 100 \%$, $100 \%$, and $87.60 \%$, respectively; in the testing phase, their accuracy was $90.16 \%, 90.16 \%, 81.97 \%, 85.25 \%$, and $88.52 \%$, respectively. Second, the performance of the SVM, KNN, Decision Tree, Random Forest, and Logistic Regression on the HF dataset during the training phase reached to an accuracy of $92.35 \%, 96.82 \%, 96.46 \%, 97.68 \%$, and $91.05 \%$, respectively; in the testing phase, their accuracy was $90 \%$, $93.33 \%, 95 \%, 95 \%$, and $88.33 \%$, respectively.

5.6. Comparison with Previous Studies. Table 15 and Figure 8 describe the evaluation of machine learning network models proposed by several criteria evaluated in relevant previous studies. As noted, previous studies were evaluated with some criteria. All previous studies reached an accuracy ranging between $93.85 \%$ and $77.55 \%$, while the accuracy of the 
proposed system reached 100\% during training and 95\% during testing. The previous studies reached a precision ranging between $91.4 \%$ and $77.4 \%$, while the proposed system reached $100 \%$ during training and $97.62 \%$ during testing. The recall (sensitivity) in previous studies reached a rate ranging between $97 \%$ and $72 \%$, while the proposed system reached $100 \%$ during training and 95.35\% during testing.

\section{Conclusion and Future Work}

The importance of electronic biometrics was verified in the process of predicting heart disease and failure. The SelectKBest function with the chi-square statistical method was applied to select the features with strong correlation with the target feature, and then, the degree between each feature and the target feature was determined. Feature engineering method was also applied to increase the number of correlated features between them and train machine learning models to obtain reliable results that were better than the results obtained from the original features of the two datasets. Machine learning algorithms used optimised hyperparameters and fed them with new features. All algorithms reached superior results during the training and testing phases of the two datasets. During the testing phase, all algorithms achieved better results for the second dataset (HF) than for the first dataset (Cleveland). For the first dataset, Random Forest and Decision Tree reached the best results during the training phase, with $100 \%$ for all measures. During the testing phase, SVM and KNN achieved better results than the rest of the algorithms. For the second dataset, Random Forest obtained the best results during both phases. There are some limitations to the study. First, the two datasets used and publicly available are relatively small. Second, the two datasets do not contain feature natriuretic peptides (NPs) which are biomarkers of heart failure, where NPs rise with age and NPs decrease in obese patients. Third, the two datasets did not include advantages about the patients' diet. However, despite the limitations, the two datasets had sufficient features. Our aim was to rank the significance of the features on the basis of the score and correlation feature of heart failure. The future scope of this work is the application of the Internet of Things and the testing of new samples in real time.

\section{Data Availability}

The data used to support the findings of this study were collected from a UCI Machine Learning Repository, in the below links: https://archive.ics.uci.edu/ml/datasets/ heart+Disease and https://archive.ics.uci.edu/ml/machinelearning-databases/00519/.

\section{Conflicts of Interest}

The authors declare that they have no conflicts of interest.

\section{Acknowledgments}

The authors would like to thank Prince Sultan University (PSU) for its support which assisted in publishing this paper.

\section{References}

[1] G. A. Roth, C. Johnson, A. Abajobir et al., "Global, regional, and national burden of cardiovascular diseases for 10 causes, 1990 to 2015," Journal of the American College of Cardiology, vol. 70, no. 1, pp. 1-25, 2017.

[2] WHO, Who-cardiovascular diseases (CVDs), 2016, January 2016, http://www.who.int/mediacentre/facts-heets/fs317/en/.

[3] Heart Rhythm Society, "Heart diseases and disorders," http:// www.hrsonline.org/Patient-Resources/Heart-DiseasesDisorders.

[4] American Heart Association, What is cardiovascular disease?, 2017, http://www.heart.org/H-EARTORG/Support/What-isCardiovascular-Disease_UCM_301852_Article.jsp\# .WgOf5VuCzIU.

[5] G. A. Roth, C. Johnson, A. Abajobir et al., "Global, regional, and national burdenof cardiovascular diseases for 10 causes, 1990 to 2015," Journal of the American Collegeof Cardiology, vol. 70, no. 1, pp. 1-25, 2017.

[6] C. Weber and H. Noels, "Atherosclerosis: current pathogenesis and therapeuticoptions," Nature Medicine, vol. 17, no. 11, pp. 1410-1422, 2011.

[7] C. Y. Karabay, G. Kocabay, A. Guler, A. Kalayci, T. Akgun, and C. Kirma, "The clinical usefulness of carotid ultrasonography in patients with an inconclusive exercise treadmill stress test result," Journal of Cardiology, vol. 64, no. 1, pp. 70-74, 2014.

[8] S. Honda, Y. Kataoka, T. Kanaya, T. Noguchi, H. Ogawa, and S. Yasuda, "Characterization of coronary atherosclerosis by intravascular imagingmodalities," Cardiovascular Diagnosis and Therapy, vol. 6, no. 4, pp. 368-381, 2016.

[9] Mayo Clinic Staff, Coronary artery disease, diagnosis, 2017, http://www.mayoclinic.org/diseases-conditions/coronaryartery-disease/diagnosistreatment/drc-20350619.

[10] I. Babaoglu, O. Findik, and M. Bayrak, "Effects of principal component analysis onassessment of coronary artery diseases using support vector machine," Expert Systemswith Applications, vol. 37, no. 3, pp. 2182-2185, 2010.

[11] D. Giri, U. R. Acharya, R. J. Martis et al., “Automateddiagnosis of coronary artery disease affected patients using LDA, PCA, ICA, anddiscrete wavelet transform," Knowledge-Based Systems, vol. 37, pp. 274-282, 2013.

[12] L. U. Zimmerli, E. Schiffer, P. Zürbig et al., "Urinary proteomic biomarkers in coronary artery disease," Molecular \& Cellular Proteomics, vol. 7, no. 2, pp. 290-298, 2008.

[13] J. H. Tan, Y. Hagiwara, W. Pang et al., "Application of stacked convolutional and long short-term memory network for accurate identification of CAD ECG signals," Computers in Biology and Medicine, vol. 94, pp. 19-26, 2018.

[14] Z. Arabasadi, R. Alizadehsani, M. Roshanzamir, H. Moosaei, and A. A. Yarifard, "Computer aided decision making for heart disease detection using hybrid neural network-genetic algorithm," Computer Methods and Programs in Biomedicine, vol. 141, pp. 19-26, 2017.

[15] S. Maji and S. Arora, "Decision tree algorithms for prediction of heart disease," in Information and Communication 
Technology for Competitive Strategies, pp. 447-454, Springer, Singapore, 2019.

[16] S. M. Saqlain, M. Sher, F. A. Shah et al., "Fisher score and Matthews correlation coefficient-based feature subset selection for heart disease diagnosis using support vector machines," Knowledge and Information Systems, vol. 58, no. 1, pp. 139-167, 2019.

[17] S. Babu, E. M. Vivek, K. P. Famina et al., "Heart disease diagnosis using data mining technique," in 2017 international conference of electronics, communication and aerospace technology (ICECA), vol. 1, pp. 750-753, Coimbatore, India, 2017.

[18] G. T. Reddy and N. Khare, "An efficient system for heart disease prediction using hybrid OFBAT with rule-based fuzzy logic model," Journal of Circuits, Systems and Computers, vol. 26, no. 4, article 1750061, 2017.

[19] M. G. Feshki and O. S. Shijani, "Improving the heart disease diagnosis by evolutionary algorithm of PSO and feed forward neural network," in 2016 artificial intelligence and robotics (IRANOPEN), pp. 48-53, Qazvin, Iran, 2016.

[20] K. Uyar and A. İlhan, "Diagnosis of heart disease using genetic algorithm based trained recurrent fuzzy neural networks," Procedia computer science, vol. 120, pp. 588-593, 2017.

[21] A. U. Haq, J. P. Li, M. H. Memon, S. Nazir, and R. Sun, “A hybrid intelligent system framework for the prediction of heart disease using machine learning algorithms," Mobile Information Systems, vol. 2018, Article ID 3860146, 2018.

[22] J. Kerexeta, A. Artetxe, V. Escolar, A. Lozano, and N. Larburu, "Predicting 30-day readmission in heart failure using machine learning techniques," HEALTHINF, pp. 308-315, 2018.

[23] E. D. Adler, A. A. Voors, L. Klein et al., "Improving risk prediction in heart failure using machine learning," European Journal of Heart Failure, vol. 22, no. 1, pp. 139-147, 2020.

[24] B. Jin, C. Che, Z. Liu, S. Zhang, X. Yin, and X. Wei, "Predicting the risk of heart failure with EHR sequential data modeling," Ieee Access, vol. 6, pp. 9256-9261, 2018.

[25] M. Gjoreski, A. Gradišek, B. Budna, M. Gams, and G. Poglajen, "Machine learning and end-to-end deep learning for the detection of chronic heart failure from heart sounds," IEEE Access, vol. 8, pp. 20313-20324, 2020.

[26] J. Vijayashree and H. P. Sultana, "A machine learning framework for feature selection in heart disease classification using improved particle swarm optimization with support vector machine classifier," Programming and Computer Software, vol. 44, no. 6, pp. 388-397, 2018.

[27] World Health Organization, World Heart DayMay 2019, https:// www.who.int/cardiovascular_diseases/world-heart-day/en/.

[28] National Heart Lung and Blood Institute (NHLBI), Heart failureJune 2019, https://www.nhlbi.nih.gov/health-topics/heartfailure.

[29] F. Meng, Z. Zhang, X. Hou et al., "Machine learning for prediction of sudden cardiac death in heart failure patients with low left ventricular ejection fraction: study protocol for a retroprospective multicentre registry in China," BMJ Open, vol. 9, no. 5, article e023724, 2019.

[30] G. A. Lewis, E. B. Schelbert, S. G. Williams et al., "Biological phenotypes of heart failure with preserved ejection fraction," Journal of the American College of Cardiology, vol. 70, no. 17, pp. 2186-2200, 2017.

[31] T. A. Buchan, H. J. Ross, M. McDonald et al., "Physician prediction versus model predicted prognosis in ambulatory patients with heart failure," The Journal of Heart and Lung Transplantation, vol. 38, no. 4, p. S381, 2019.
[32] B. Chapman, A. D. DeVore, R. J. Mentz, and M. Metra, "Clinical profiles in acute heart failure: an urgent need for a new approach," ESC heart failure, vol. 6, no. 3, pp. 464-474, 2019.

[33] J. Gallagher, D. McCormack, S. Zhou et al., "A systematic review of clinical prediction rules for the diagnosis of chronic heart failure," ESC heart failure, vol. 6, no. 3, pp. 499-508, 2019.

[34] E. M. Senan and M. E. Jadhav, "Classification of dermoscopy images for early detection of skin cancer-a review," International Journal of Computer Applications, vol. 975, p. 8887, 2019.

[35] M. A. Aleid, K. A. Alyamani, M. Rahmouni, T. H. Aldhyani, N. Alsharif, and M. Y. Alzahrani, "Modelling the psychological impact of covid-19 in Saudi Arabia using machine learning," Computers, Materials \& Continua, vol. 67, no. 2, pp. 20292047, 2021.

[36] https://archive.ics.uci.edu/ml/datasets/heart+Disease.

[37] https://archive.ics.uci.edu/ml/machine-learning-databases/ 00519/.

[38] E. M. Senan, F. W. Alsaade, M. I. A. Al-mashhadani, H. H. Theyazn, and M. H. Al-Adhaileh, "Classification of histopathological images for early detection of breast cancer using deep learning," Journal of Applied Science and Engineering, vol. 24, no. 3, pp. 323-329, 2021.

[39] G. T. Reddy, M. P. K. Reddy, K. Lakshmanna, D. S. Rajput, R. Kaluri, and G. Srivastava, "Hybrid genetic algorithm and a fuzzy logic classifier for heart disease diagnosis," Evolutionary Intelligence, vol. 13, no. 2, pp. 185-196, 2020.

[40] M. S. Amin, Y. K. Chiam, and K. D. Varathan, "Identification of significant features and data mining techniques in predicting heart disease," Telematics and Informatics, vol. 36, pp. 82-93, 2019.

[41] S. Pouriyeh, S. Vahid, G. Sannino, G. De Pietro, H. Arabnia, and J. Gutierrez, "A comprehensive investigation and comparison of machine learning techniques in the domain of heart disease," in 2017 IEEE symposium on computers and communications (ISCC), pp. 204-207, Heraklion, Greece, 2017.

[42] D. Chicco and G. Jurman, "Machine learning can predict survival of patients with heart failure from serum creatinine and ejection fraction alone," BMC Medical Informatics and Decision Making, vol. 20, no. 1, p. 16, 2020. 